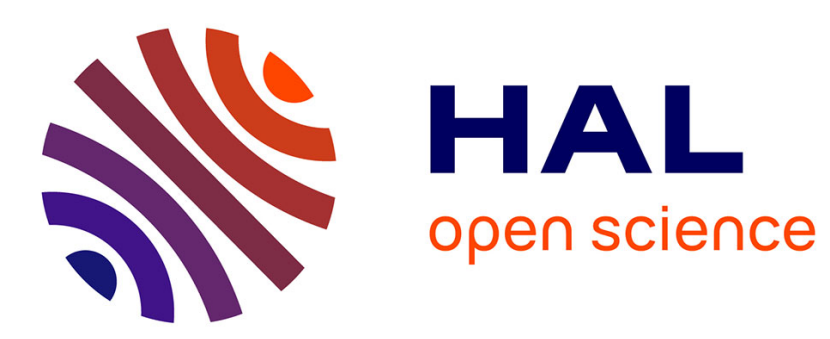

\title{
Cosmic Evolution and Universal Evolutionary Principles Leonid Grinin
}

\section{To cite this version:}

Leonid Grinin. Cosmic Evolution and Universal Evolutionary Principles . From Big Bang to Nanorobots , pp.20-45, 2015. hprints-01830789

HAL Id: hprints-01830789

https://hal-hprints.archives-ouvertes.fr/hprints-01830789

Submitted on 5 Jul 2018

HAL is a multi-disciplinary open access archive for the deposit and dissemination of scientific research documents, whether they are published or not. The documents may come from teaching and research institutions in France or abroad, or from public or private research centers.
L'archive ouverte pluridisciplinaire HAL, est destinée au dépôt et à la diffusion de documents scientifiques de niveau recherche, publiés ou non, émanant des établissements d'enseignement et de recherche français ou étrangers, des laboratoires publics ou privés. 


\title{
I. UNIVERSAL EVOLUTIONARY PRINCIPLES
}

\section{1 \\ Cosmic Evolution and Universal Evolutionary Principles}

\author{
Leonid E. Grinin
}

\begin{abstract}
The present article attempts at combining Big History potential with the potential of Evolutionary Studies in order to achieve the following goals: 1) to apply the historical narrative principle to the description of the star-galaxy era of the cosmic phase of Big History; 2) to analyze both the cosmic history and similarities and differences between evolutionary laws, principles, and mechanisms at various levels and phases of Big History. As far as I know, nobody has approached this task in a systemic way yet. It appears especially important to demonstrate that many evolutionary principles, patterns, regularities, and rules, which we tend to find relevant only for higher levels and main lines of evolution, can be also applied to cosmic evolution. Moreover, almost everything that we know about evolution may be detected in the cosmic history, whereas many of the evolutionary characteristics are already manifested here in a rather clear and salient way. Of course, many of the characteristics are manifested in initial or nonsystematic forms but some features, on the contrary, appear to be more distinct just in the cosmic phase. And at the same time when many characteristics and features which are typical of biological and social evolution unexpectedly reveal their roots or protoforms at earlier phases, one becomes aware that the universal character of evolution is real and it can be detected in a number of manifestations. One should also bear in mind that the origin of galaxies, stars, and other celestial objects is the lengthiest evolutionary process among all evolutionary processes in the Universe. Such an approach opens new perspectives for our understanding of evolution and Big History, of their driving forces, vectors, and trends, it also creates a consolidated field for the multidisciplinary research.
\end{abstract}

Keywords: Star-Galaxy Era, cosmic phase of Big History, laws of evolution, universal evolutionary principles, Universe, preadaptations, Evolutionary Studies, evolutionary selection, additive and substitutive models of evolution, largescale structures of Universe, gas-dust clouds, non-uniformity concentration of matter, circulation of matter in the Universe, dark and light matter.

Evolution: From Big Bang to Nanorobots 2015 20-45 


\section{THE FORMATION OF THE LARGE-SCALE STRUCTURE OF THE UNIVERSE}

The formation of modern structure of the Universe lasted for many billions of years when our Universe 'lived' for quite a long period of time without any stars, galaxies, Hubble's law, clusters and superclusters of galaxies (Khvan 2008: 302). Now it is recognized that the first stars and galaxies turn out to have emerged much earlier. According to the latest astronomical observations, the first galaxies emerged not later than several hundred million years after the Big Bang. In what follows we will consider this in detail. What was the matter from which they had emerged?

\section{Tiny Material for the Formation of Giants: About the Gas-Dust Clouds and Cosmic Dust}

Approximately 270,000 years after the Big Bang, a large phase transition occurred resulting in the emergence of matter in the form of atoms of hydrogen and helium. Later, they started to consolidate in new structures. The main mass of this matter concentrated in gas-dust clouds that could be of tremendous sizes (dozens parsecs, or even more). ${ }^{1}$ At present we usually speak of such cosmic fractions as interstellar gas and cosmic dust. They can be both in vacuum condition and in the form of clouds. But as is known the observed today clouds consist mainly of equal proportions of gas and dust. That is why they are usually called gas-dust clouds.

For the first time we observe Nature in the role of a constructor. Before that, it had formed just the basic elements. Now one could observe the emergence of enormous structures from tiny particles and 'specks of dust'. Later one could constantly observe similar processes in evolutionary developments: large-scale structures are composed of myriads of minute particles and grains.

Minor factors are also necessary for structuring. The formation of clouds (and later of stars and galaxies) involved concentrating of matter on enormous scale, which could have been caused only by gravity. However, this only force is insufficient for structuring, because in 'an absolutely homogenous universe the emergence of large-scale structures (galaxies and their clusters) is impossible' (Dolgov et al. 1998: 12-13). Thus, certain 'seed grains' are needed, similar to the process of formation of rain drops emerging around particles of dust or soot; or the formation of a pearl around grit.

Small fluctuations are often needed for the powerful forces to start working. Actually, minor fluctuations (minute deviations from homogeneity and isotropy) occurred in the Universe from the first nanoseconds after the Big Bang. Then the larger fluctuations happened. They could act as seed grains for the formation of galaxies. However, it is not clear what kind of fluctuations caused the formation of galaxies and what the mechanism of their formation is. In other fields of evolution initial fluctuations also often remain a mystery.

\footnotetext{
${ }^{1} 1$ parsec $\approx 31$ trillion $\mathrm{km}$.
} 
Thus, the non-uniformity (including the non-uniformity connected with different concentration) is one of the main foundations of development and evolution at all its stages and in all its forms. Any major evolutionary shift in biological and social matter at a certain stage of evolution is necessarily connected with some form of accumulation or concentration when matter becomes abundant and occupies certain niches (the periods which are similar to the first stages after the Big Bang). The higher the stage of evolution, the more important it is. Thus, in a large-scale system the common processes may proceed in their usual way, whereas in the concentration zone some peculiar processes start (as it takes place in the stellar formation zones).

\section{The Epoch of Formation of the Large-Scale Structure of the Universe. First Galaxies and Stars}

Dark and light matter. Nowadays it is generally accepted that dark matter plays an important role in the formation of the first galaxies, as it appeared capable of much quicker consolidation into clusters than the light (baryonic) matter. The latter could not condense until the end of the hydrogen recombination (atom formation) due to radiation pressure (270,000 years after the Big Bang). Only when hydrogen nuclei and electrons were able to merge and form atoms, whereas photons separated from the matter and flew away, the radiation pressure dramatically decreased to zero. As a result, the light matter would fall in potential holes prepared by the dark matter. Perhaps, we observe here a very interesting evolutionary pattern. Nevertheless, the non-evolutionary dark matter initially appeared to be more capable to structuring than the light matter, but the progress of the former toward structuring turned out to be very short and almost leading to a dead-lock. However, as with any evolutionary dead end, this does not mean an absolute stagnation. At present, in galaxy halos the dark matter continues structuring in certain smaller structures, the so-called clumps and sub halos (see, e.g., Diemand et al. 2008). Meanwhile, the evolutionary potential of the light matter was based on the 'achievements of the dark matter'. Such a model of development is rather typical for evolution. For example, long before the transition to agriculture some gatherers of cereal plants invented many things (including tools, granaries, and grinding stones) that later turned to be rather useful for agriculturalists, but the hunter-gathering mode still turned out to be an evolutionary dead end.

There are rather diverse opinions on the timing and characteristics of the process and sequence of formation of stars, galaxies, galaxy clusters and superclusters.

The galaxy protoclusters are supposed to have been the first to originate. As Ph. J. E. Peebles (1980: 389-390) notes, 'The same process could operate on a larger scale, the first generation of gas clouds being protoclusters that fragmented to form galaxies, some clusters dissolving to produce field galaxies. A sequence of this general sort has appealed to many authors'. Such phenomena take place at higher levels of evolution when something general is formed 
(which will turn into a larger taxon in future) that later differentiates into primary level taxa. The species and classes in biology form in this way. The same refers to a society: at first there emerge rather large formations such like families of languages and then the languages, ethnic super-groups and then ethnoses, and sometimes large early empires or states; and afterwards within their framework statehood goes one or two levels down. In other words, there emerges a non-differentiated large structure which is capable to produce a great number of peculiar structures.

However, a more commonly held hypothesis suggests that protogalaxies (in the form of giant condensed gas clouds) were the first to emerge within the structure of the Universe, and later they became the birthplace for individual stars and other structural elements (see, e.g., Gorbunov and Rubakov 2012: 27). However, in recent years new evidence has come to hand to support the idea that those were the stars that appeared first. This discovery somehow modified the previous theories. As a result, at present it is widely accepted that the stars were first to emerge, but those were the giant stars, much more massive than most of the later-formed ones (May et al. 2008). Because of the absence of carbon, oxygen and other elements that absorb the energy from condensing clouds, the process proceeded more slowly in that epoch; thus, only giant clouds could condense producing massive stars hundreds times larger than the Sun (Ibid.). Nowadays there are also such giants of 100-200 solar masses but they are considered unstable (see Surdin and Lamzin 1992). We will see below that the larger is star, the shorter is its life. Thus, such giant stars lived only a few million years. In addition, the first stars contained a small amount of heavy elements. Thus, more than one generation of stars could change, until the quantity of heavy elements gradually increased. The emergence of 'heavy elements' from the 'dead star stellar remnants' resembles the formation of fertile soil from the remnants of dead plants. The circulation of matter in the Universe is always observed everywhere and at all levels.

In recent years we have witnessed the discovery of a few galaxies that are claimed to be the oldest in the Universe. Meanwhile, the dates of formation of the first galaxies are shifted closer and closer to the Big Bang. The emergence of the first galaxies is dated to less than 400 million years after the Big Bang; and there are even claims that some more ancient galaxies have been discovered. They are claimed to have emerged only 200 million years after the Big Bang. ${ }^{2}$

The evidence on the first stars refers to $c$. 150-200 million years after the Big Bang; hence, stars and galaxies appear to have emerged almost simultaneously. Since that time depending on its density the matter in the Universe coexists in three main types: in dense state in celestial bodies, in rarefied state in the clouds of different size, and in low-density state (in tens of times compared to the clouds) in interstellar gas.

\footnotetext{
${ }^{2}$ The evidence refers to the team of the French astronomer Johan Richard who seemed to discover the galaxies that formed 200 billion years after the Big Bang (see European Commission 2011).
} 


\section{THE ERA OF THE STAR-GALAXY STRUCTURE OF THE UNIVERSE}

The formation of galaxies and their clusters, as well as of stars and other celestial bodies was the longest evolutionary process that had ever taken place in the Universe. At present we observe that this process is still going on alongside changes and disappearance of galaxies and stars. During the first eight billions years, the formation of huge diversity of stellar bodies and new heavy elements took place in the Universe until about 5-4.5 billion years ago there the conditions were formed for the formation of stellar (Solar) system. On one of its planets there started new geological, chemical and biochemical processes.

\section{The Structure of the Universe in the Past and Present}

Evolutionary principles of the structure of the Universe. Thus, the formation of the large-scale structure of the Universe has not occurred at once. Formation of galaxies and their clusters, probably, was the process which had lasted for billions years.

There are several evolutionary principles in the characteristics of the structure of the Universe which are well traced at all levels of evolution. But we will consider only two of them.

1. The combination of antagonistic features. In the structure of the Universe one can find the combination of uniformity and non-uniformity. The uniformity is already manifested at the inflation phase, when the Universe started inflating evenly in all dimensions. The uniformity has preserved till present, but only at the largest scale (of an order of magnitude of 100 megaparsecs). For reference, the size of the largest galaxy clusters (such as our Local Group with the center in the Virgo constellation) is 40 megaparsecs at most (Gorbunov and Rubakov 2011). The nonuniformity of the Universe is manifested at scales smaller than 100 megaparsecs; and the smaller is the scale, the more salient is the non-uniformity. The combination of antagonistic features is a phenomenon that is rather characteristic for many other evolutionary levels. Thus, the antagonistic features of 'even surface' and 'uneven surface' are quite applicable to the Earth surface: at bird's eye it looks even.

2. Density and sparsity can be traced everywhere, starting from the atomic structure, where the mass is concentrated in a tiny nucleus, while most of the atom is an empty space. There is a huge non-uniformity between the scale of the Universe and the space that the main mass of (at least, baryonic) matter occupies within it. At the present stage of evolution of the Universe its matter is concentrated, first of all, in stars which actually occupy only a $10^{-25}$ part of the total volume of the Universe (not taking into account the galaxy nuclei [Pavlov 2011: 43]). Were there such proportions in ancient Universe? Maybe, not. Therefore, the concentration of the matter is strengthening. Not only the hard matter is distributed very unevenly throughout the Universe; the same is true of the gas. Much of this gas is concentrated in giant molecular clouds which are of many thousands of solar masses (Lipunov 2008: 37). At the same time the difference in density is fractal, which is especially evident in the zones of high 
density. The factors contributing to such unevenness are not always clear; for example, it is not clear, what the uneven distribution of masses during the formation of galaxies (Weinberg 1975: 608) as well as many other processes of distribution, concentration and dissipation are connected with. But the principles of uneven distribution of the matter mass at different evolutionary levels are rather similar. For example, at present the main mass of the Earth's population is concentrated in a rather small territory in comparison with the total territory where life on the Earth is possible.

The structure of the contemporary Universe. The main structural elements of the Universe are galaxies, their clusters, and superclusters. Our Metagalaxy is mainly formed of superclusters. ${ }^{3}$ All the structural elements are rather stable in terms of gravitation, though they can split, merge, and collide, etc.

Galaxies are integral structural entities with a rather complex structure which includes, in addition to regions arms, etc., a nucleus (core), semiperiphery (so called 'disc'), and periphery (so called 'halo') (Baade 2002: 255). The halo consists of both single stars and various stellar clusters. The halo's radius (a few hundred thousand light years) is much larger than the radius of the galaxy's disc. ${ }^{4}$

A galaxy generally contains from 100 to 200 billion stars. There are small (dwarf) galaxies with a few million stars, as well as giant galaxies consisting of up to a trillion stars.

Our Galaxy with the mass of about $10^{11}$ solar masses is one of the giants. However, the mass of our neighbor - the Great Andromeda Nebula (M31, is found in our cluster of galaxies (the Local Group) - is about three times larger. Probably, the most famous M87, which is situated in the central part of the cluster of galaxies in Virgo constellation, has the largest mass. Apparently, the mass of this Galaxy exceeds by hundreds times the mass of our Galaxy. At the other pole there are dwarf galaxies whose masses are $\sim 10^{7}$ of solar mass, that is several dozens of times more than the mass of globular cluster (Shklovsky 1987: Part 1, Chapter 6).

Stars are distributed rather unevenly throughout galaxies, stars are parts of various groups and clusters; some of them consist of just a few stars, but some clusters can contain up to several million stars. For example, they identify more than 1,500 star clusters in our Galaxy (Surdin and Lamzin 1992). There are many globular clusters, which are the spherical clusters tightly bound by gravity and consisting of hundreds of thousands, which occupy the spherical volume in the space. These are rather old stars (there are about 150-200 such clusters in our Galaxy). According to Hubble, the galaxies are classified into spiral, ellip-

\footnotetext{
${ }^{3}$ If, according to some researchers, Metagalaxy is not the only one in the Universe, then for some time it will be considered as the largest structural unit of the Universe (see Pavlov 2011: 52). If the Universe is not a Universum but a so-called Multiverse, then the Universes or their groups will be the maximum unit of the structure.

${ }^{4}$ There might be an invisible halo consisting of dark matter behind the visible halo. It may be found in many (if not all) galaxies, whereby the diameter of the dark halo might exceed the diameter of the visible halo by an order of magnitude (see Ryabov et al. 2008: 1131).
} 
tical, and irregular with various subtypes (Baade 2002: 18-32); yet, by now one more galaxy type has been identified - the lenticular galaxies. The latter make about 22 per cent among our nearest galaxies. In these galaxies a bright main flattened body, 'a lens', is surrounded by a weak halo. Sometimes the lens is surrounded by a ring (see Novikov 1979: Chapter $1, \S 8$ ). More than a half of the members of the Local Group are the older elliptical galaxies with intermediate or minor luminosity (Ibid.: 31). Galaxies are complex and to a considerable extent self-regulating systems, within which there is a constant circulation of matter: some stars disintegrate, whereas new stars emerge from cosmic gas and dust. Thus, the circulation (which results in the processes of renovation of matter and its mixing) takes place at all its spatial levels and at different levels of evolutional complexity.

An average galaxy cluster usually consists of 500-1000 galaxies. Galaxy clusters have a rather regular structure which is likely to include a massive nucleus in the center. Galaxy superclusters are entities consisting of 2-20 galaxy clusters and galaxy groups as well as of isolated galaxies. In general, there are known more than 20 superclusters, including our Local Group.

Generations of galaxies and stars. There are rather diverse opinions not only on the time of formation of stars and galaxies but also on the number of generations throughout the evolution of the Universe. In addition, there is no consensus on which galaxies should be regarded as old, and which galaxies should be considered young. The point is that within a single galaxy one can find stars and their aggregates which considerably differ in their type, age, and other parameters. Yet, it appears possible to single out a few widely accepted basic ideas.

1) In the evolution of the Universe, there have been three (or at least two) generations of galaxies and stars. In general, old galaxies are smaller and dimmer. Their stars contain dozens of times smaller quantities of heavy elements than the Sun. The astronomers can hardly observe any star formation processes within such galaxies. There is also a hypothesis that more dark mass is concentrated in old galaxies in comparison with younger ones. The same way, older and younger stars differ from each other in their size, luminosity, and chemical composition. ${ }^{5}$

2) One can hardly define an exact periodization of generations of galaxies, because the process of formation of galaxies and stars is constantly going on. Galaxies need to constantly renew their composition in order to retain their identity. As Joseph Shklovsky maintains, in this respect galaxies are very similar to primary forests with its mix of tree ages (whereas the age of trees is much

\footnotetext{
${ }^{5}$ The later the star was formed, the more heavy elements (the remnants of interstellar nuclear reactions) it includes. This has become one of the arguments in favor of multistage star formation in the galaxy (Surdin and Lamzin 1992).
} 
less than the age of the forest in general [Shklovsky 1984: 45]). ${ }^{6}$ The mobility and variability of the celestial landscape resembles very much the mobility of geological landscapes.

One should also consider the fact that the formation of galaxies can proceed in different ways, for example, through the absorption of smaller galaxies by the massive ones when the collision between the galaxies takes place. 'If a small galaxy comes into collision with a massive one, it is absorbed by it and loses its identity; and every time when passing near a massive galaxy the stars estrange from it' (May et al. 2007: 140). In this case both young and old clusters and groupings of stars are combined (see about it below). Another pattern is through merging. Galaxies of younger generations can sometimes form through the accretion of a few small, weak and compact galaxies into a single galaxy. In this case they became 'building blocks' for the present galaxies. Finally, it may happen that two massive galaxies collide. Such a collision may take billions of years and be accompanied with active star formation and emergence of massive and very bright stars. The latter means that these are short-living stars, that is there will be many bursts of nova and supernova. Finally, galaxies may diverge again, but in this case they turn out to be very different from what they used to be before the collision, whereas one more galaxy may emerge out of the matter estranged from the both galaxies (see May et al. 2008: 142).

One can find numerous analogies to those models of galaxy formation in biological, geological, and, especially, social evolution. As stars and galaxies are composed of more or less homogenous matter (which can be divided or united rather easily), they somehow paradoxically resemble societies that consist of people who can be included into other societies through integration or capture. On the other hand, captures are also attested among social animals, for example among ants.

4) Galaxies are collections of different types of stars. However, there are certain peculiarities as regards the position of old and young stars within galaxies which is probably connected with the self-regulation within the galactic systems or the peculiarities of star formation which occurs in large groups, or with other factors. Thus, within our galaxy the younger stars (such as the Sun which is a few billion years old) are generally larger, hotter and brighter. They are located closer to the disc plane, and, especially, within the galaxy arms; whereas in the galaxy periphery (in its halo) one can find older stars more than 12 billion years old (which suggests the overall age of our galaxy). Yet, of course, older and younger stars may be also located rather close to each other. Thus, one may find many old stars near the galaxy center (bulge), but there are also young stars that emerged from the matter produced by the disintegration of

\footnotetext{
${ }^{6}$ Though, according to Shklovsky, the age of small stars can exceed the age of the galaxy (it is the large stars that die quickly); therefore, he supposes that in the course of time the number of small stars will increase (Shklovsky 1984: 45).
} 
older stars. The highest stellar density is found in the galaxy center where it reaches a few stars per cubic parsec.

As we mentioned, the very old, not bright, not hot and not massive stars contain many times less amounts of heavy elements than the Sun. It is not surprising that these stars and young, hot, and bright stars of the surface of disc, arms and halo were named 'Population I' and 'Population II'. ' It is generally accepted that the majority of globular clusters are very old (12 billion years old or even more). But the dispersed or open galactic clusters are dozens or even hundred times younger than globular clusters (i.e. they are just hundreds of millions of years). But there are younger stellar associations (see, e.g., Surdin and Lamzin 1992; Surdin 2001).

On the one hand, the preservation of generations of stars and galaxies demonstrates an additive character of the evolution of abiotic systems; however, the capture of stars and galaxies with their subsequent integration and prolonged processes of collision of galaxies demonstrates that in abiotic natural systems one may also find some other models of evolution - connected with 'wars' and 'submission of outsiders'.

The type of development through the emergence of different generations of individuals and species (preserving certain generic features, on the one hand, and accumulating important changes in their structure and characteristics, on the other) is rather widespread at all phases and levels of universal evolution. Within any biological class or order (e.g., perissodactyls) we can show how important characteristics vary and gradually change from one species to another, whereas due to those characteristics some species press out others and occupy better niches (see, e.g., Grinin, Markov, and Korotayev 2008). Various types of states and civilizations also rather vividly illustrate the progress: for example, more organized and developed states emerge through the absorption of the achievements of less developed generations of states, which one can illustrate using examples from the history of Ancient Rome, Byzantium, some Medieval European states and so on. The coexistence of different generations sometimes leads to the situation when younger and more advanced entities either transform the older ones or form a symbiosis with them (though in some places one may find 'restrictions' for older types and generations).

\section{Change of the Chemical Composition of the Universe}

Though, hydrogen has always been the most abundant element in the Universe chemical composition; its share constantly decreased. This occurred (and occurs) because hydrogen is the main fuel for the nuclear fusion reactions that support life and luminosity of stars. Despite tremendous amounts of released energy during these reactions, the energy release rate is very low. For example,

\footnotetext{
${ }^{7}$ This is an outdated division of stars. That is why when there appeared an evidence of the existence of the stars of the first generation which emerged at the age of the Universe of 150 million years from the moment of the Big Bang (see about it above), they were named 'Population III' in order to follow the conventional designation.
} 
the intensity of the solar radiation is $2 \mathrm{erg} / \mathrm{g} \cdot \mathrm{s}$, which is almost equal with the piles of burning leaves. Stars shine brightly because they are massive and large (Surkova 2005: 9).

Increasing temperatures inside the core of some stars were needed for the formation of new elements that were absent in the era of recombination. However, all the fusion reactions that occur to produce elements larger than iron no longer release energy. Reactions of another type are needed for the formation of elements heavier than iron - those reactions consume more energy than release. That is why there are such relatively small amounts of heavy elements in the Universe. Yet, such peculiar reactions do take place - for example, in neutron stars and during explosions of supernovas. In supernovas, during their bursts, for about just 100 seconds heavy elements form, which expand Mendeleev's periodic table including uranium and thorium (Ibid.).

When a supernova explodes, heavy elements are expelled through the Universe with stellar winds and through the fall of the dispersed matter on the surface of cosmic bodies (the so-called accretion). As stars turn to be the main centers of the synthesis of chemical elements, the distribution of heavy elements in the Universe is very inhomogeneous.

The emergence of heavy elements and their concentration in certain bodies and compositions are extremely important processes, which lead to an enormous increase in the number of matter combinations, and consequently have an evolutionary potential; in particular, they lead to the start of the full-scale chemical, biochemical, and biological processes. In certain respects, such a slow and uneven accumulation of new structural elements (heavy elements) resembles the process of an accumulation of valuable mutations in biological evolution, or the accumulation of valuable innovations in social evolution (all of them expand the evolutionary potential and increase the rates of evolutionary changes).

The similarities and differences of stars in their structure (the presence of heavy elements) are similar to the similarities and differences in genome. All living organisms mainly have the similar structure and all huge differences are caused by small divergences (by several percent) in genes.

\section{The Evolution of Galaxies and Stars}

Processes of the formation of galaxies and stars. Until quite recently, the processes of star formation were entirely concealed from an external observer; however, at present due to the technological progress one can observe some aspects of those processes in many parts of our galaxy. Those observations confirm the theory of stellar formation from cold clusters which are heated by gravitation and pressure.

On the whole, this process may be described in the following way. Within giant hydrogen and helium clouds, some heterogeneities emerge (which is quite natural for atmosphere) which launch (under certain conditions) the gravitation processes that start to collect that mass into spherical forms. Sometimes a direct formation of a giant mass of gas clouds takes place, from which a galaxy or a 
star cluster later emerges. In this case the cloud fragmentation may occur and thus, more and more gas-cloud spheres (there could be hundreds of millions, or even hundreds of billions of them) emerge, which can gradually transform into protostars. This process continues up to the point when the gas density becomes so high that each new fragment already has a mass of a star (Surkova 2005: 49). Then the gravity starts impeding further fragmentation. This process is denoted as 'a cascade fragmentation'. It is remarkable that it resembles certain processes in social evolution - for example, the fragmentation of large early states into separate parts that decentralize up to the point when further division becomes unreasonable (e.g., in certain periods there were dozens and hundreds of independent states in the territories of Germany or France).

As enormous gas/dust clouds appear unstable, they disintegrate into large bundles, so the formation of stars proceeds in groups. This phenomenon is of interest not only with respect to stellar evolution. The group formation is rather typical for evolution in general (in this way populations and sometimes new species emerge; chiefdoms, city-states, and sometimes political parties emerge in groups, and so on).

The process of the further star formation is connected with the point that the initial compression heated the gas to a rather high temperature that, on the one hand, prevents the further compression of the gas, and, on the other hand, eventually contributes to the onset of the nuclear fusion reaction (Hawking 2001: 63-64).

Diversity of stars and galaxies. Diversity is an absolutely essential prerequisite of evolutionary development. And this condition is fully realized within cosmic evolution. Stars greatly differ in their mass, temperature, luminosity, age and the lifetime. They also differ in many other characteristics including the chemical composition and the immediate system to which they belong, for example whether they are binary or isolated stars, whether the stars have the planet system or not, etc. Those differences may vary greatly.

The differences in influence of gravitation and the peculiarities in behavior under its influence of gas-cloud masses could become the reason of formation of different types of galaxies. That means that the galaxies are born either as spiral or elliptic ones and in the course of evolution the type of galaxy is preserved. A galactic structure is to a large degree determined by the initial conditions of its formation (e.g., by the character of rotation of the original gas clump from which a galaxy is formed).

The sizes and mass of stars vary greatly. For example, with respect to masses, stars range in mass from about 0.1 to 100 or more solar masses. There are some considerations that the thermonuclear fusions cannot go in a star with the mass of less than $8 \%$ of the Solar mass that is why such objects do not belong to the stars. The stars of small masses prevail. Thus, for every ten million of red dwarfs there are only about a thousand of giants and only one supergiant. It is rather natural that the number of smaller entities is orders of magnitude larger; actually, the same phenomenon may be observed, for example, in Zool- 
ogy or Political Geography where the number of small animals or countries is much larger than that of large ones.

Individual fates within evolution. Individualism of stars is exceptionally diverse, despite the fact that the parameters in which they differ are limited. Thus, one can maintain that with the formation of stars we observe the emergence of individual objects in nature, 'individuals' that, on the one hand, are rather similar, but, on the other hand, have rather different individual fates much depending on circumstances of their birth and various contingencies. For example, stars with small masses (in which nuclear fusion occurs at a slow rate) can use all of their fuel (i.e., remain in the main sequence) for many billions of years, the potential lifetime of some stars can exceed the modern age of the Universe. On the other hand, blue giants (which quickly consume fuel and lose most part of their mass due to their instability) burn out hundreds of times faster.

The stars end their lives in a rather different way. Some of them, having lost one or a few outer layers, would cool, slowly transforming into cold bodies; some others may contract a few dozen times, or may end their lives with huge explosions blowing their matter into open space; the latter outcome may be considered as a kind of altruism. Finally, a star may become a black hole that does not allow any matter to come out of its immensely compressed depths.

\section{Stellar Birth, Stages of Life and Death}

Protostars. As mentioned above, stars can emerge through the condensation and compression of gas clouds under the influence of gravitational forces. This is a protostar phase. In comparison with the subsequent life of a star, the period of its slow contraction seems rather short; however, actually this is not a quick process as it continues sometimes up to 50 million years (Surkova 2005: 50). During this period of time, there is a tremendous rise of temperature at the core of the protostar, the temperature may grow up to 8-10 million Kelvin, and, as a result, thermonuclear reactions become possible. The protostar becomes a young star. However, an external observer will only be able to see it in a few hundred thousand (or even a few million) years when the cocoon of gas and dust envelope the protostar dissipates.

Actually, there happens a miracle: a giant shining incandescent body, which is capable of living for billions of years, emerges from an absolutely amorphous, lacking any structure, opaque, and cold mass of gas mist. In other words, we deal here with a vivid example of self-organization that takes place under the influence of gravitation and thermodynamic laws. In particular, an intensive contraction leads to heating, which increases the internal pressure, which, eventually, stops the compression process.

One may also note that the emergence of stars and galaxies must have a certain trigger that creates turbulence and heterogeneity. Those triggers (catalyzers) are the inherent components of evolutionary mechanisms that may be found in many processes: in chemical and geological processes, within biological evolution with respect to fast formation of species, or within social evolu- 
tion with respect to state formation (see Grinin 2011, 2012 for more details). The supernova shock wave, the collision of a molecular cloud with spiral arms of a galaxy and other events can become such a trigger to the star formation (Surkova 2005: 50).

Another (and the longest) macrophase is the main sequence star. During this phase of the stellar lifetime, nuclear-fusion reactions of burning hydrogen to helium in the core, keep the star shining. That is why the duration of the main sequence phase depends mainly on the stellar mass. The more massive the star, the shorter is its lifespan on the main sequence (as with a larger mass the 'fuel combustion' processes run more intensively). A star preserves its size and form due to the mutual struggle of two forces: the gravity that tries to compress the star and the gas pressure produced as a result of nuclear reactions and powerful heating. There is a dynamic equilibrium between temperature and gas pressure. With growing temperature, the gas expands and works against the gravitation forces, which results in cooling of the star; this way the thermal balance is kept. In the lifetime of stars and galaxies, as well as at all other levels of evolution, we find numerous cases and different forms of the interaction between two opposite processes which make it possible for 'individuals' to live. The processes of assimilation and dissimilation support vital activities within biological organisms; the processes of animal reproduction and their extermination by predators support the population balance; interaction between processes of production and consumption is the basis of the reproduction of social systems, and so on.

Red giants. The new phase of stellar evolution is connected with the exhaustion of hydrogen supplies. The gas pressure (that maintained the star balance when necessary fuel was available) decreases and the stellar core compresses. This leads to a new increase in temperature. A star starts to burn heavier elements and thus, the stellar composition significantly changes. Simultaneously with the compression of the core, the star's outer layers expand. In general, the star inflates and expands a few hundred times, and it transforms into a red giant and then with the larger expansion - into a red supergiant (large stars with the mass of more than ten solar masses transform into supergiants at once). This phase lasts for about one tenth of the 'active lifetime' of a star, when the processes of nuclear fusion go on in its depths.

Star death: three cases. The next phase is the transformation of a red giant or supergiant. Actually, the new form depends on stellar mass and a number of other characteristics such as the stellar rotation and velocity, the degree of its magnetization, and so on. The following three outcomes are considered most typical. They depend on stellar mass (but the limit value estimates vary significantly, and so below I will mention the main alternative values after the slash). ${ }^{8}$

\footnotetext{
${ }^{8}$ According to one of classifications (that might be more correct than the one reproduced below), one can subdivide all the stars just into two classes: massive stars (with a mass equal or exceeding c. 10 solar masses), producing neutron stars and black holes, and non-massive ones producing white dwarfs (Lipunov 2008: 99). But the latter ones, depending on the mass, differ in the elements which form their core.
} 
Stars with the masses smaller than 1.2-1.4/3 solar masses transform from red giants into the so-called 'white dwarfs', when the star sheds its outer envelope to form a planetary nebula with an extremely contracted core (down to the size of the Earth). The further compression does not occur because of the so-called degenerate electron gas pressure that does not depend on temperature. As a result, the white dwarf is rather stable. However, due to the lack of hydrogen and helium, thermonuclear fusions can no longer proceed within such a star. A white dwarf is very hot when it is formed; yet, afterwards the star cools and transforms into a 'black dwarf', that is, it becomes a cold dead cosmic body.

For stars with initial mass of more than $1.2-1.4 / 3 M_{\odot}$, but less than $2.4-$ $3 / 7-103 M_{\odot}$, their slow and gradual aging results in an 'infarct', that is a collapse. After the depletion of hydrogen and the decrease of the internal gas pressure (that used to balance the gravity), under the influence of gravity the core gets extremely compressed (by dozens thousand times - up to the radius of ten kilometers) just in a few seconds. Almost simultaneously the outer layers of the star are blown away with a huge speed as a result of shock wave. This supernova shines brighter than millions of ordinary stars, but for a very short period of time. This explosion expels the stellar material into interstellar medium and thus, there occurs the formation of considerable quantities of heavy (heavier than iron) elements that afterwards concentrate in various celestial bodies. The remaining core contracts to become a neutron star (which is supposed to contain super dense neutron fluid). With respect to its size, such a star is five billion times smaller than the Sun, but it is hundreds of thousands of times brighter because the temperature on its surface is 1000-1500 times higher than on the Sun (Lipunov 2008: 133).

If stellar mass exceeds the limit of $3 / 7-10 M_{\odot}$, after hydrogen is burnt out it will start collapsing and explode (though sometimes it may collapse without an explosion), but the force of compression will be unlimited, as the gravity becomes enormous because of the huge mass and absence of internal forces that can prevent the collapse. The action of the gravitational force which is balanced by nothing leads to the situation when the stellar diameter becomes infinitesimally small. According to theoretical calculations, the star is transformed into a black hole whose gravity fields are strong for light to escape.

\section{UNIVERSAL EVOLUTIONARY PRINCIPLES THAT APPEARED DURING THE STAR-GALAXY ERA \\ Life, Death, and Catastrophes in the Evolutionary Aspect}

The irreversible character of evolution is its most important characteristic. It can be observed as a steady movement to more complex structures and forms of organization, to the changes in the chemical composition of the Universe, etc. As regards the individual objects, the irreversible character of evolution is obvious and undoubted. A star which passed through a certain phase of life cannot reenter this phase. 
The problem of the individual's death. Death as an opportunity for life to go on. Stellar life and death can hardly leave anybody indifferent. Actually, within the Big History framework, this is the first time when we come across the problem of a life cycle of individual objects in such an explicitly expressed form. On the one hand, the star's fate, lifespan, and type of death depend on initial parameters, as if they were 'genetically programmed' (and, hence, they may be forecasted); on the other hand, they may be altered by some contingencies. Thus, the star's fate is not 'fatal', indeed. Binary star systems increase highly the variability of the individual star fates; as Lipunov (2008: 252) puts it, we deal here with a kind of 'quadratic evolution'. What is more, it is actually possible to speak about differences in the 'individual' stellar behavior or 'within a group', because the interaction of two, three, and more stars may lead to very significant differences and unusual results that cannot emerge within the development trajectory of individual stars. In fact, similar patterns are observed at other levels of evolution, when behavior of pairs or groups of individuals produces outcomes radically different from the ones observed with respect to the behavior of an individual not interacting with others.

Finally, the meaning of individual's death for evolution may be different. Up to a certain degree one may observe a direct correlation between the 'strength' of death, the power of the stellar explosion, and the formation of conditions for a new evolutionary search. Stellar explosions affect the dynamics of their environment; consequently, they may help create unusual conditions that contribute to the emergence of certain developmental deviations. Within tens of thousands years the zone of explosion expands to a vast area of interstellar medium (covering the distances of dozens of parsecs); in this area one can see the formation of new physical conditions (in particular, temperature, density of cosmic rays and magnetic fields strength). Such a disturbance enriches the respective zone with cosmic rays and brings changes to chemical composition (Shklovsky 1984: 209). The explosions also contribute to star formation. Thus, a star does not die in vain. One can draw here an interesting analogy with extinctions in biological evolution which contribute to new directions of speciation. The stellar destruction can be also compared with the disintegration of large empires with all the subsequent repercussions. The disintegration of a large empire leads to a cascade of new states forming both in the place of the empire and even beyond its borders. Historical detonation contributes to politogenesis the same way as the cosmic detonation contributes to star formation.

\section{Stellar life in terms of self-organization and maintaining of the dynam-} ic equilibrium. In the initial phase under the compression a cloud of gas 'burns' itself like packed straw or rags mow burn. The next phase of selforganization is connected with the formation of complex stellar structure on the main sequence phase during which burning out of hydrogen occurs. After burning out of the most part of hydrogen a star enters a new phase, it expands and transforms into a red giant. At the same time the processes of self-organization occur again and the stellar structure radically changes (highly compressed core 
coexists with the expanded envelopes). After the fuel is burnt out in a red giant, the next phase is compression under the influence of the gravitational force and formation of a brand-new structure: small but very massive core with extremely high density of the matter within it.

Let us consider the stellar life in terms of maintaining and breaking the equilibrium. First of all, there is a thermal equilibrium, when the rate of energy produced in the core (through thermonuclear fusions) balances the loss of energy through the emission of radiation into space. This equilibrium is broken when hydrogen fuel is gone. The reserves are apparently compensated when a star starts using another type of energy. This may occur through the contraction of the star which begins fusing helium into carbon, thus producing many times more energy for every atom; afterwards heavier elements may be used as fuel, and each heavier element will produce more and more energy per atom. Meanwhile, the core of the star begins to increase in temperature. There is equilibrium in terms of pressure of different forces and preservation of a certain form and size of the star. Within the main sequence phase, the balance is maintained as the gravity pulls all the stellar matter inward, toward the core, while gas pressure pushes heat and light away from the center. This pressure exists until the reserves of nuclear fuel are exhausted (Efremov 2003: 97). With respect to red giants one may speak about equilibrium of another kind in two dimensions. In the core the temperature grows due to contraction and thermonuclear reactions of higher levels start (i.e., involving not hydrogen but helium or heavier elements). As a result of those reactions the temperature may grow up to 100 million Kelvin. That is why a stronger gravity is balanced by a stronger (due to temperature) gas pressure. In the meantime, within the shell the equilibrium is achieved through the multifold expansion of the outer layers. In neutron stars and white dwarfs, the subsequent phases of the stellar lifetime, there is their peculiar equilibrium.

Structuring, self-organization and 'Russian nesting doll' structure. The whole history of star-galaxy phase of cosmic evolution is the history of formation of different structures of different size and grouping of these structures into larger ones. At the same time, as we already mentioned, we deal here with the ability of objects to self-organization at all phases of general and individual evolution. It is very important that structuring occurs not only among stars and galaxies but also among molecular clouds. The latter can be regarded as a parallel branch of evolution. Parallelism plays a great role in evolution dramatically increasing the opportunities of transition to something new and creating a field of contacts between various directions of evolution (see about it below).

Giant molecular clouds as a rule have a rather complex 'Russian nesting doll' structure when small and large condensations are nested into larger and more vacuum ones (see Surkova 2005: 48). 'Russian nesting doll' structure (strongly resembling a fractal one) is also typical of higher levels of evolution. Thus, smaller groups of herding and social animals, which are the part of larger groups, are similar in a general way to the structure of a large society. This also 
refers to social evolution, in particular, the organizations which are not centralized, for example, tribal alliances. The components of the latter (lineages, clans, subtribes) are less similar to the structure (and the principle of structuring) of a tribe. That is why the tribes can easily be separated and if necessary be gathered. This is typical of the amalgamations of the representatives of the fauna (flocks, herds).

Synthesis of gradualism and catastrophism. With respect to cosmic evolution one may observe a combination of two principles that provoke endless discussions in geology and biology. The subject of those discussions is what principle prevails in evolution. Are we dealing mostly with slow gradual changes, eventually leading to major changes (gradualism)? Or, does the development mostly proceed through sharp revolutionary breakthroughs which in biology are often connected with catastrophes? Within star-galaxy evolution the combination of both principles is more than just evident. Here, as at no other evolutionary level, both patterns of evolution are organically combined in individual fates of the stars. The main sequence phase of stellar evolution (when the fusing of hydrogen occurs) demonstrates the gradual character and the importance of slow and prolonged processes. However, catastrophes of various scales can take place within the lifetime of any star. For some stars, such radical changes may manifest in major - but still local - changes (such as shedding the outer layers), whereas for other stars these might be tremendous catastrophes when stars die, figuratively speaking, 'brightly' and 'heroically', illuminating the Universe, leaving a billion-year-long footprint of light. The latter, that is the extraordinary phenomena and events, both among the stars and among humans are fewer than the former, that is the common ones.

\section{Some Evolutionary Ideas in Connection with the Star-Galaxy Phase of Evolution of the Universe}

In the evolutionary process (and also as a whole in cosmic evolution) of formation of stars, galaxies, nebulae, and cosmic clouds one can distinguish a number of important evolutionary principles and laws that are not evident. Their detection is important for understanding the unity of principles of development of the Universe. Those principles and observations are grouped into several blocks.

- Evolution proceeds via constant creation and destruction of objects. Nature, when creating, destroying, and renewing various objects, 'tests' many versions, some of which turn out to be more effective and have more chances to succeed in terms of evolution. For such a situation of selection within constant destruction and creation process, it appears possible to apply a rather appropriate notion of 'creative destruction' introduced by Josef Schumpeter (2007).

- 'Evolution is stronger than individual objects'. Cosmic processes are accompanied with constant emergence, development, change, and death of various objects (stars, galaxies, and so on). Thus, here one can point as relevant the principle that was expressed by Pierre Teilhard de Chardin (1987) with respect 
to life in the following way: 'life is stronger than organisms', that is, life goes on exactly because organisms are mortal. The same is relevant to stellar evolution. We may say here that the cosmos is stronger than stars and galaxies; and in general, evolution is stronger than individual objects.

- Rotation and keeping balance take place due to constant destruction (or transition to new phases in the lifecycle) of some objects and the emergence of the others. This keeps balance and creates conditions for development, because development is a result of change of generations and species.

- In every end there is a beginning. Star-evolutionary 'relay race'. The material of dead objects becomes building blocks for the formation of new objects. This represents the circulation of matter and energy in nature; on the other hand, this represents a sort of 'relay race'. The latter allows using the results of long-lasting processes, in particular, the accumulation of heavy elements (for example, the Solar System was formed from the remnants after the explosion of a supernova; that is believed to be one of the reasons of the presence of great number of heavy and super-heavy elements on the Earth and other planets). ${ }^{9}$ Thus, we deal here with the above mentioned 'creative destruction' - the creation of new objects due to the destruction of the old ones. Furthermore, the new objects are different from the old ones, and sometimes these differences are quite apparent. It ensures continuity and provides new forms with space for advancement (e.g., the change of generations of biological organisms always results in certain transformations). The change of rulers may not necessarily lead to radical social changes; however, each new ruler is somehow different from his predecessor, as a result the accumulation of historical experience occurs.

- New generations of organisms and taxa are the ways of qualitative development. One may also detect generations of taxa, which already have significant evolutionary and systemic differences. Thus, generations of stars differ in terms of their size, chemical composition, and other characteristics. Only through the change of several generations of objects this class of objects acquires some features that, nevertheless, are considered to be typical for the whole class of objects. (Thus, species in biology are determined by the impossibility to sire with the representatives of other species. However, many species reproduce asexually).

\section{Individuality as a way to increase evolutionary diversity}

- Ontogenesis and phylogenesis. The evolution proceeds at various levels: through the development of its certain branch, a certain class, species and so on (and sometimes even at the level of an individual organism). Besides, if apply biological terminology, at every level of evolution we find a combination of processes of ontogenesis and phylogenesis. Of course, within star-galaxy evolution the phylogenesis is represented much weaker than in the evolution of life. Nevertheless, it still appears possible to speak about the history of trans-

\footnotetext{
${ }^{9}$ About the rule of evolutionary relay race see Grinin et al. 2008.
} 
formation of certain types of galaxies and stars, and, hence, up to a certain extent the cosmic phylogenesis does occur (see as above with respect to change of a few generations of stars and galaxies that differ from each other as regards their size, structure, and composition).

- The phases of individual development (ontogenesis) - myriads of different paths. Every type of objects has their own regular phases of life which depend on both internal characteristics of the object and the environment (proximity of other objects, etc.). As we have already seen, stars depending on their mass, composition and other characteristics have very different duration of the phase which is called the main sequence (from several tens of millions of years to $10-15$ billion of years and even more). As was mentioned above, the fate of stars at the last stage of their life also depends on their mass and other circumstances. Depending on this they can turn into the White Dwarf, become a neutron star or a Black Hole.

- Required and excessive variation as conditions of a search for new evolutionary trajectories. Within the processes described above one can observe the formation of the taxonomic diversity of the objects; we may even speak about occupying the evolutionary 'niches'. There emerge the types of stars which have different mass, luminosity (accordingly, different spectrum/color of the light), temperature, system (single stars, planet systems and systems of stars from two to seven), period of rotation, magnetic field, etc. The same refers to the galaxies among which one can distinguish a number of types (elliptical, spiral, and lenticular) and subtypes. Such diversity is extremely important. Only the achievement of a necessary level of taxonomic and other diversity allows a search for ways to new evolutionary levels. This is sometimes denoted as the rule of necessary and excessive diversity (see Grinin et al. 2008: 68-72; for more details see also Panov 2008).

- Norm, averages, and deviation from a norm. Only when we find a sufficient diversity, it appears possible to speak about norm, average level, exceptions, and outliers. Scientists have long known that the breakthroughs to new forms usually happen somewhere at a distance from the former main directions, at the periphery (see the next part about the structure), and in those systems that diverge from the previous mainstream.

- Continuity, which actually means the emergence of a continuum of forms, sizes, life spans, and lifecycles, is rather characteristic for space objects. Thus, the stars can be presented as a continuum from heavier to lighter ones, whereas the latter become hardly distinguishable from planets, their temperature does not contribute to the thermonuclear reactions, etc. The types of planetary systems uniformly cover a wide range of parameters. There is also a sequence of phases in the transformation of cosmic clouds into stars: condensation of clouds - formation of protostars - formation of young stars, and up to the death of stars. A wide range (a continuum) of forms and sizes of objects may be observed at geological, biological, and social phases of the evolution. 


\section{Object, environment, competition, development systems, and self-preservation}

- The relations between structure and environment. Multilevel systems (galaxy - galaxy cluster - galaxy supercluster) act as a system of a higher order for stars, and, simultaneously, they create an environment that produces an enormous influence on those stars. A star directly interacts with its immediate environment (e.g., with neighboring stars because of the strong gravity which affects the movement of both stars), whereas with the distant environment the interaction proceeds at its higher levels. Within star-galaxy evolution the environment generally produces less impact than at other evolutionary levels but nevertheless, it is highly important. For example, the role of the immediate environment is very important in systems of double, triple, or multiple stars. On the whole, single stars are separated by great distances and that is why they collide rather infrequently except for the center of the galaxies where star density is much higher. There occurs one collision once a million years (Shklovsky 1987: Ch. 1). For a small galaxy the influence of neighboring larger galaxy may turn out to be fatal, if it leads to its absorption. A star explosion close to clouds may (as we have seen) trigger the process of formation of stars and galaxies. The role of the environment is important for planets; the most important thing of this environment will be characteristics of a star and nearest planets as well as the influence of satellites and the danger of collisions.

With the development of a certain type of evolution, its own laws and environment gain a growing influence on the development of its subjects and actors. For example, both abiotic nature and the biotic environment influence biological organisms. However, within a complex ecological environment, it is the intraspecies and interspecies competition that may have larger influence than any other natural factors, whereas within a complex social environment it is just the social surrounding that affects individuals and social systems more than the natural forces do (though in consuming societies the role of influence of natural environment on people is much more important). Thus, with the formation of star-galaxy structure of the Universe there emerged macro-objects which start to interact with environments which are larger by many orders of magnitude.

- The formation of evolutionary driving forces of development. The study of cosmic evolution shows that evolutionary driving forces emerge just at this phase of evolution (although they turn to have small-scale impact on 'progress'). Of course, evolutionary changes are determined by the influence of physical or chemical forces, but we observe them sometimes in the form of preadaptations. For example, the emergence of organic chemical compounds in the clouds of molecular gas exemplifies such a preadaptation. In principle such kinds of complex compounds do not play a significant role in cosmic evolution, but they are in the 'reserves' of development. It is of interest that a peculiar type of structure of such clouds which protect the molecules from cosmic radiation, makes their existence possible. In other words, special conditions are required for preadaptations. Preadaptations in biology often emerge in special 
environment. Thus, it is supposed that the transformation of crossopterygian fin, which gave rise to amphibians, into a limb occurred in terms of shrinking shallow water.

- 'Struggle' for preservation of forms. It is important to note that stars, galaxies and planets (as well as other celestial bodies) have their definite, quite structured, and preserved form. The 'struggle' for the preservation of those forms, the capacity to live and shine, the use of different layers to minimize energy losses lead to a slow but evident evolutionary development. This way the atomic composition of the Universe changes, whereas the diversity of variations of the existence of matter increases. The bilateral transition of matter to atomic (in hot bodies) or molecular state (in cold structures, in particular in the clouds of gas and on outer layers of stars) and vice-versa when forming from the giant clouds of stars is an outstanding manifestation of this type of evolution, a preparation for the formation of its biochemical and biological forms.

- The urge toward self-preservation and origins of the struggle for resources. The emergence of structures that strive for their preservation (as mentioned previously) creates a wide range of interaction between the system and its environment; on the other hand, this creates a basis for the "evolutionary search' and evolutionary advancement. This evolutionary paradox, namely, that the struggle for the self-preservation is the most important source for development, can be observed here in its full-fledged form. However, the star-galaxy evolution demonstrates the emergence of this driving force which will become very important in biological evolution; and it appears to be the most important driving force in social evolution. This is the struggle for resources that among stars and galaxies may proceed in the form of weakening of another object or its destruction (e.g., through a direct transfer of energy and matter from one body to another, i.e. accretion), in the form of 'incorporation', 'capturing', that is 'annexation' of stars and star clusters by larger groups. We have already mentioned above galactic coalescences. Thus, some astronomers maintain that throughout a few billions of years our galaxy has 'conquered, robbed, and submitted' hundreds of small galaxies, as there are some evident 'immigrants' within our galaxy, including the second brightest star in the northern sky, Arcturus (Gibson and Ibata 2007: 30). It is widely accepted that emergence and expansion of a black hole may lead to the 'eating' of the matter of the nearby stars and galaxies. However, the 'eating capacity' of the black holes is greatly exaggerated in popular literature but this is quite excusable as black holes are very mysterious objects. In systems of double stars or in star-planet systems one may also observe such a form of interaction as the exchange of energy and resources.

- External factors as the triggers of transformations play a great role, for example passing by giant molecular clouds of a large celestial object, stellar explosion, etc. can start the process of stars and galaxies formation (i.e. become the trigger of gas concentration). Collisions between celestial bodies can cause the creation of new objects. Thus, it is supposed that the Moon was formed as a result of a collision of a large object with the Earth. 


\section{Multilinearity}

Multilinearity is one of the most important characteristics of evolution.

Unfortunately, it does not get sufficient attention, and there is a tendency to reduce evolution to a single line - the one that has produced the highest complexity level, which is often interpreted as the main line of evolution. However, at every stage of evolutionary development one can find an interaction of a few lines that can have rather different futures. In other words, in addition to the main evolutionary line one can always identify a number of lateral ones. Firstly, they contribute to the increasing diversity; secondly, they allow expanding the range of search opportunities to move to new levels of development; thirdly, the lateral lines may partly enter the main evolutionary stream, enriching it. We quite often deal with two or more coexisting and comparable lines of development whose convergence may lead to a quantitative breakthrough and synergetic effect. Various lines of development may transform into each other. Elsewhere we have written a lot on the issue of social evolution in this context (see, e.g., Korotayev et al. 2012; Grinin 2011).

- Classical forms and their analogues. The main and lateral lines of evolution may be considered in two dimensions: 1) horizontal (as regards complexity and functions), 2) vertical (concerning the version that would be realized later at higher evolutionary phases). It appears also possible to speak about classical versions and their analogues. Thus, various forms of aggregation and specialization of unicellulars can be regarded as analogues of multicellulars (see Eskov 2006), whereas various complex stateless polities can be regarded as state analogues (see Grinin and Korotayev 2009; Grinin 2011, 2012 for more details). Classical forms and their analogues can transform into each other; however, these are just the analogues that tend to transform into classical forms, rather than the other way round (the latter may be regarded both as a direct degeneration and as a forced adaptation to sharply changing conditions). ${ }^{10}$

- Stars and molecular clouds: two parallel forms of existence of cosmic matter. In this respect we may consider stars and galaxies as the main line of evolution and the giant clouds as its lateral lines; also the former may be designated as 'classical forms', and the latter may be defined as 'analogues'. In fact, on the one hand, galaxies and stars emerge from giant molecular clouds. On the other hand, as we have seen, these clouds have the same gravitational force and even structural complexity as stars and galaxies ${ }^{11}$ have. And they are also able to concentrate, to take part in the energy exchange, etc. They also exceed the stars in the level of organization of elementary particles as the molecules are concentrated in the clouds, and there is the concentration of elemen-

\footnotetext{
${ }^{10}$ Thus, in difficult environmental conditions, (e.g., of semi-deserts and deserts), centralized forms of polities - large chiefdoms and early states - can dissolve into a system of interacting societies and polities but without centralization (see Grinin and Korotayev 2009, 2011; Korotayev 2000).

${ }^{11}$ Accordingly, on different levels of generalization the clouds of one size are the analogues of the stars and the clouds of larger size - of galaxies.
} 
tary particles and atom's nuclei in the stars. ${ }^{12}$ Besides, stars when losing the matter, shedding its envelopes and through the explosion transform into gasdust clouds, i.e. into interstellar gas which forms the molecular clouds.

\section{INSTEAD OF CONCLUSION. The Formation of Various Evolutionary Lines at the Microworld Level}

Astrophysical and astrochemical evolution. Almost from the very beginning of the development of the Universe (when the temperature reached thousands of Kelvin) chemical evolution emerges as accompanying physical and astrophysical evolution. Of course, chemical evolution also occurs within stars with the emergence of heavier elements. However, that was rather laying the foundations for chemical evolution, because chemical processes involve reactions which lead to the emergence of new substances. Such processes proceed, first of all, within gas-dust clouds where molecules emerge. Hydrogen molecules are absolutely prevalent in number; however, molecules of water and some other substances also emerged. Chemical evolution goes on also on planets (where it combines with geological, or rather planetary evolution) as well as on small celestial bodies (asteroids and meteorites, etc.). At the same time on the planets where due to volcanism, pressure upper layers to lower ones and other geological processes the temperatures could be high enough, chemism significantly differed from that in cold clouds.

The position of chemical evolution in cosmic evolution. The representatives of dialectical materialism following Friedrich Engels (in his 'Dialectics of Nature' [Engels 1940]) stated (and we can agree with this statement) that the chemical form of organization of matter is evolutionarily higher than physical. In contrast with biological and social forms which from their very start displayed substantially higher levels of organization of the matter, the chemical form (that emerged a rather short time after the physical form) did not represent a higher form of evolution for a rather long period of time. The same refers to the geological form which emerged on the planets long time ago but it became higher only after the formation as a result appropriate conditions. That is not to say that chemical evolution is not important in the framework of general stellar and galactic evolution; however, before the emergence of the Earth-like planet, the physical and chemical forms of organization of matter should be regarded as equally important, constantly transforming into each other (see also Dobrotin 1983: 89). ${ }^{13}$ The chemical form of development in many respects was regarded as the 'preadaptation' for new levels of evolution. Let us note that in biology the term 'preadaptation' denotes the situation in which such achievements do not play the important role as a whole (without taking account of the certain organ-

\footnotetext{
${ }^{12}$ The molecules can also be found in periphery layers of some stars, in the places with low temperatures.

${ }^{13}$ In any case, it is important to note that due to thermonuclear reactions the chemical evolution of the Galaxy proceeds in a single direction, namely, from simple to complex elements (Surdin and Lamzin 1992). This also refers to evolution in general.
} 
ism) in the environment where they emerged. But without them it appears impossible to make a breakthrough at a certain moment. As a result, at some evolutionary turning point the forms which have these 'preadaptations' benefit from this and become evolutionarily higher or leading. They can give an impulse to the formation of new taxa and occupying new ecological niches. Within the Big History framework, the principle of 'preadaptation' means that at the level where a preadaptation emerges, it generally plays insignificant role; however, at a new evolutionary level such innovations generally give evolutionary impulses. ${ }^{14}$

\section{References}

Baade W. 2002. Evolution of Stars and Galaxies. Moscow: URSS. In Russian (Бааде В. Эволюция звезд и галактик. М.: УРСС).

Diemand J., Kuhlen M., Madau P., Zemp M., Moore B., Potter D., and Stadel J. 2008. Clumps and Streams in the Local Dark Matter Distribution. Nature 454(7205): 735-738.

Dobrotin M. N. 1983. Dialectics and Problems of the Development of the Chemical Form of the Matter Movement. Dialectics of Nature and Natural-Science Pattern of the World. Materialistic Dialectics: 5 vols. Vol. 3. Chapter III, pp. 73-91. Moscow. In Russian (Добротин М. Н. Диалектика и проблемы развития химической формы движения материи. Диалектика природы и естественно-научная картина мира. Материалистическая диалектика: в 5 т. Т. 3, гл. III, с. 73-91. M.)

Dolgov A. D., Zeldovich Ya. B., and Sazhin M. V. 1998. Cosmology of the Early Universe. Moscow: Moscow University Press. In Russian (Долгов А. Д., Зельдович Я. Б., Сажин М. В. Космология ранней Вселенной. М: Издательство Московского Университета).

Efremov Yu. N. 2003. Deep into the Universe. Moscow: URSS. In Russian (Eфpeмов Ю. Н. Вглубь Вселенной. М.: Едиториал УРСС).

Engels F. 1940. Dialectics of Nature. New York: International Publishers.

Eskov K. Yu. 2006. Amazing Paleontology. History of the Earth and Life on It. Moscow: Enas. In Russian (Еськов К. Ю. Удивительная палеонтология. История Земли и жизни на ней. М: Энас).

European Commission - MEMO 2011. EU Marie Curie researcher discovers galaxy 13 billion light years away. URL: http://europa.eu/rapid/press-release_MEMO-11237_en.htm.

Gibson B., and Ibata R. 2007. The Phantom of Dead Galaxies. V mire nauki, June: 2935. In Russian (Гибсон Б., Ибата Р. Призраки погибших галактик. В мире науки, июнь: 29-35).

Gorbunov D. S., and Rubakov V. A. 2011. Introduction to the Theory of the Early Universe: Cosmological Perturbations and Inflationary Theory. Singapore - Hackensack, NJ: World Scientific Publishing Company.

\footnotetext{
${ }^{14}$ About the preadaptations in the megaevolution framework, see Grinin, Korotayev, and Markov 2011.
} 
Gorbunov D. S., and Rubakov V. A. 2012. Introduction to the Theory of the Early Universe: Hot Big Bang Theory. $2^{\text {nd }}$ ed. Moscow: LKI. In Russian (Горбунов Д. С., Рубаков С. А. Введение в теорию ранней Вселенной. Теория горячего Большого взрыва. 2-е изд. М.: ЛКИ).

Grinin L. E. 2011. The Evolution of Statehood. From Early State to Global Society. Saarbrücken: Lambert Academic Publishing.

Grinin L. E. 2012. Macrohistory and Globalization. Volgograd: Uchitel.

Grinin L. E., and Korotayev A. V. 2009. The Epoch of the Initial Politogenesis. Social Evolution and History 8(1): 52-91.

Grinin L. E., and Korotayev A. V. 2011. Chiefdoms and Their Analogues: Alternatives of Social Evolution at the Societal Level of Medium Cultural Complexity. Social Evolution and History. Special Issue. Chiefdoms: Theories, Problems, and Comparisons 10(1): 276-335.

Grinin L. E., Korotayev A. V., and Markov A. V. 2011. Biological and Social Phases of Big History: Similarities and Differences of Evolutionary Principles and Mechanisms. Evolution: A Big History Perspective / Ed. by L. E. Grinin, A. V. Korotayev, and Barry H. Rodrigue, pp. 158-198. Volgograd: 'Uchitel' Publishing House.

Grinin L. E., Markov A. V., and Korotayev A. V. 2008. Macroevolution in Wild-Life and Society. Moscow: LKI. In Russian (Гринин, Л. Е., Марков, А. В. Коротаев А. В. Макроэволючия в живой природе и обществе. М.: Издательство ЛКИ).

Hawking S. 2001. A Brief History of Time. From the Big Bang to Black Holes. St. Petersburg: Amfora. In Russian (Хокинг С. Краткая история времени. От большого взрывва до черных дыр. СПб.: Амфора).

Khvan M. P. 2008. Furious Universe. From Big Bang to Accelerating Expansion, From Quarks to Superstrings. Moscow: LKI. In Russian (Хван М. П. Неистовая вселенная. От Большого взрыва до ускоренного расширения, от кварков до суперструн. М.: ЛКИ).

Korotayev A. V. 2000. From State to the Chiefdom? From Chiefdom to Tribe? (Some General Tendencies of Evolution of the South Arabian Socio-Political Systems over the Past Three Thousand Years.) Early Forms of the Social Organization. Genesis, Functioning, Historical Dynamics / Ed. by V. A. Popov, pp. 224-302. SaintPetersburg: Museum of Anthropology and Ethnography Named after Peter the Great (The Kunstkamera Museum) of the Russian Academy of Sciences. In Russian (Коротаев А. В. От государства к вождеству? От вождества к племени? (Некоторые общие тенденции эволюции южноаравийских социальнополитических систем за последние три тысячи лет.) Ранние формы сочиальной организации. Генезис, функиионирование, историческая динамика / Ред. В. А. Попов, с. 224-302. СПб.: Музей антропологии и этнографии им. Петра Великого (кунсткамера) РАН.)

Korotayev A. V., Bondarenko D. M., and Grinin L. E. 2012. Social Evolution: Alternatives and Options (To the Problem Statement). Universal and Global History (Evolution of the Universe, Earth, Life and Society) / Ed. by L. E. Grinin, I. V. Ilyin, and A. V. Korotayev, pp. 348-377. Volgograd: Uchitel. In Russian (Коротаев А. В., Бондаренко Д. М., Гринин Л. Е. Социальная эволюция: альтернативы и варианты (к постановке проблемы). Универсальная и глобальная история 
(эволючия Вселенной, Земли, жизни и общества). Хрестоматия, ежегодное издание / Ред. Л. Е. Гринин, И. В. Ильин, А. В. Коротаев, с. 348-377. Волгоград: Учитель).

Lipunov V. M. 2008. In the World of Binary Stars. Moscow: LIBROKOM. In Russian (Липунов В. М. В мире двойных звезд. М.: ЛИБРОКОМ).

May B., Moore P., and Lintott C. 2008. Bang: The Complete History of the Universe. Baltimore, MD: Johns Hopkins University Press.

Novikov I. D. 1979. Evolution of the Universe. Moscow: Nauka. In Russian (Новиков И. Д. Эволючия Вселенной. М.: Наука).

Panov A. D. 2008. Cohesion of Social and Biological Evolution and the Limit of Its Acceleration. Historical Psychology and Sociology of History 2: 25-48. In Russian (Панов А. Д. Единство социально-биологической эволюции и предел ее ускорения. Историческая психология и сочиология истории 2: 25-48).

Pavlov A. N. 2011. Geophysics. The General Course on the Nature of the Earth. St. Petersburg: Russian State Hydrometeorological University. In Russian (Павлов А. Н. Геофизика. Общий курс о природе Земли. СПб.: РГГМУ).

Peebles P. J. E. 1980. The Large-Scale Structure of the Universe. Princeton: Princeton University Press.

Ryabov V. A., Tsarev V. A., and Tskhovrebov A. M. 2008. Search for Dark Matter. Uspekhi fizicheskikh nauk 178(11): 1130-1164. In Russian (Рябов В. А., Царев В. А., Цховребов А. М. Поиски частиц темной материи. Успехи физических наук 178(11): 1130-1164).

Schumpeter J. A. 2007. Theory of Economic Development. Capitalism, Socialism and Democracy. Moscow: Eksmo. In Russian (Шумпетер Й. Теория экономического развития. Капитализм, сочиализм и демократия. М.: Эксмо).

Shklovsky I. S. 1984. Stars: Their Birth, Life and Death. $3^{\text {rd }}$ ed. Moscow: Nauka. In Russian (Шкловский И. С. Звезды: их рождение, жизнь и смерть. Изд. 3-е. М.: Наука).

Shklovsky I. S. 1987. Universe, Life, Mind. $6^{\text {th }}$ ed., revised. Moscow: Nauka. In Russian (Шкловский И. С. Вселенная, жизнь, разум. 6-е изд., доп. М.: Наука).

Surdin V. G. 2001. Stars Production. Moscow: URSS. In Russian (Сурдин В. Г. Рождение звезд. М.: УРСС).

Surdin V. G., and Lamzin S. A. 1992. Protostars. Where, How and Out of What Stars Have Been Formed. Moscow: Nauka. In Russian (Сурдин В. Г., Ламзин С. А. Протозвезды. Где, как и из чего формируются звезды. М.: Наука).

Surkova L. P. 2005. Stars and Stellar Clusters in Our Galaxy. Chita: Zabaikalsky State University. In Russian (Суркова Л. П. Звезды и звездные группировки в нашей Галактике. Чита: ЗабГПУ).

Teilhard de Chardin P. 1987. The Phenomenon of Man. New York: Harper Perennial Modern Classics.

Weinberg S. 1975. Gravitation and Cosmology. Principles and Applications of the General Theory of Relativity. Moscow: Mir. In Russian (Вайнберг С. Гравитация и космология. Принципь и приложения общей теории относительности. М.: Мир). 
